\title{
Communications
}

\section{TARMED Suisse}

\section{Décisions de la CPI}

Les nouvelles décisions CPI prises durant l'été 2008 sont répertoriées ci-après. Vous trouverez la liste de toutes les décisions en vigueur sur le site officiel www.tarmedsuisse.ch $\rightarrow$ TARMED $\rightarrow$ Interprétation CPI $\rightarrow$ Décisions de la CPI $\rightarrow$ Version actuelle 1.42 du 9.9.2008.

Information concernant la «Nouvelle prestation de TARMED Suisse»: désirez-vous être informé/e automatiquement, en permanence et gratuitement des nouvelles décisions CPI? Dans l'affirmative, veuillez envoyer un courriel directement à Madame Sylvia Luca, du siège de TARMED Suisse (sylvia.luca@tarmedsuisse.ch), pour qu'elle vous inscrive sur la liste de distribution.

Les décisions CPI en lien avec le RE III relatif au chapitre 39 sont mentionnées dans la présente liste uniquement par leur titre.

\section{Décisions CPI (sans le chapitre 39)}

08010.2 A-H: Formations approfondies en pédiatrie

(endocrinologie-diabétologie pédiatrique / gastroentérologie pédiatrique / cardiologie pédiatrique / néphrologie pédiatrique / oncologiehématologie pédiatrique / neuropédiatrie / pneumologie pédiatrique / rhumatologie pédiatrique)

\section{Valable dès le: 11.6.2008}

Domaine de validité: cf. listes selon document officiel sur www.tarmedsuisse.ch.

Décision: à la recommandation de la PaKoDig, la CPI décide que les «médecins titulaires d'une formation approfondie pédiatrique ou en pédiatrie peuvent fournir les positions tarifaires indiquées dans le domaine de validité en tant que prestations en plus des positions tarifaires qui leur sont autorisées sur la base de leur «valeur intrinsèque qualitative» et les facturer au répondant des coûts.
08011: Changement de DCl, test fonctionnel conforme aux directives

Valable dès le: 7.5.2008

Domaine de validité: 17.1630

Décision: lors de la mise en place d'un défibrillateur cardioverteur implantable (DCI), le test fonctionnel standard est déjà indemnisé par la position tarifaire 17.1630 .

\section{4: Consilium}

\section{Valable dès le: 11.6.2008}

Domaine de validité: 00.2110

Décision: lorsque des discussions interdisciplinaires concernant des patients ambulatoires sont menées dans le cadre de colloques oncologiques hospitaliers internes (Tumor Boards), les prestations du médecin traitant hospitalier ne peuvent pas être indemnisées en tant que consilium, pour autant qu'un consilium n'ait pas été demandé explicitement par un médecin externe à l'hôpital...

08015: Interprétation tarifaire 04.03.04 et 04.03.01

Valable dès le: 3.7.2008

Domaine de validité: 04.03.04, 04.03.01

Décision: il convient d'appliquer le principe selon lequel la surface à facturer concerne l'activité qui est indemnisée par la (les) position(s) correspondante(s) ...

08017: Travail en l'absence du patient (y compris l'étude du dossier)

Valable dès le: 10.3 .2008

Domaine de validité: 00.0140, 02.0070, 02.0160, 02.0260, 02.0360

Décision: vaut comme étude du dossier l'étude de documents tiers relatifs au patient (lecture et évaluation de documents détaillés externes et non pas de ceux rédigés par le médecin et figurant déjà dans le dossier médical du patient) ... Demeure réservée comme exception motivée l'étude extensive de ses propres documents. Ceci doit être documenté et mis à la disposition de l'assureur à sa demande ... La présente décision remplace les interprétations I-04043 et I-07012.
08040: Règles de cumul pour les prestations additionnelles

Valable dès le: 11.6.2008

Domaine de validité: tout le tarif

Décision: IG-6 et IG-9: sauf indication contraire, les règles de cumul concernant une prestation principale sont applicables aussi pour les prestations additionnelles associées à cette prestation principale

08044: 15.0190, interprétation de courbes spirométriques

Valable dès le: 3. 7.2008

Domaine de validité: 15.0190

Décision: le texte français et italien de la position 15.0190 ne correspond pas au texte allemand déterminant et n'est pas appliqué dans ces régions linguistiques conformément au tarif ...

08048: Rapport médical sur formulaire

Valable dès le: 9.9.2008

Domaine de validité: 00.0010, 00.0140, 00.2285

Décision: l'article 6, alinéa 4 OPAS (Ergométrie): ... L'établissement d'une ordonnance est indemnisé dans le cadre d'une consultation ou comme prestation en l'absence du patient; si l'assureur demande un rapport détaillé, celui-ci pourra être facturé avec la position 00.2285 ss.

08049: Interprétation 00.0840

Valable dès le: 9.9.2008

Domaine de validité: 00.0840

Décision: la position tarifaire 00.0840 est facturable au max. $1 \times$ par ponction, indépendamment du nombre de préparations injectées. 
Décisions CPI concernant le chapitre 30 TARMED, corrections résultant de RE III

- 08021: corriger le texte 39.3550

- 08022: corriger le texte et introduire une nouvelle interprétation dans la position 39.3560

- 08023: corriger le texte 39.3570

- 08024: introduire l'interdiction de cumul entre 39.0011 et 39.0021

- 08025: corriger le texte et l'interprétation 39.1330

- 08026: corriger l'interprétation 39.3420, supprimer la règle régissant la quantité

- 08027.1: règles concernant la quantité et les pages

- 08028: supprimer l'indication «sur la même page» pour les interdictions de cumul 39.4070 et 39.4080

- 08029: introduire le type de traitement 39.3820

- 08030: arthrographie des articulations

- 08031: corriger IG-45

- 08032: supprimer l'interdiction de cumul entre 39.3240 et 39.3280 de même qu'entre 39.3260 et 39.3280 . Corriger l'interprétation 39.3260

- 08033: supprimer l'interdiction de cumul 39.3270 avec le chapitre 39.03.02.02 et introduire une nouvelle interdiction de cumul avec 39.3265

- 08034: corriger les interprétations 39.3408 et 39.3404 , la valeur intrinsèque additionnelle dans 39.3404

- 08035: corriger le texte GP-33, corriger et compléter l'unité fonctionnelle (F et I) dans IG-47

- 08036: corriger l'interprétation 39.0050 et 39.0055

- 08037: corriger IC-39.05-1

- 08038: corriger IC-39.04-1

- 08039: supprimer BP-20, nouvelles règles de cumul, nouveau texte BP-53

- 08050: limitation des quantités Prestations de base techniques, patient ambulatoire chapitre 39

- 08051: limitation des quantités Consultation de base/unité d'exploitation, radiologie à l'institut de radiologie de l'hôpital et à l'institut de radiologie à l'extérieur de l'hôpital

\section{SGAM}

20. Zürcher Workshop «Allgemeinmedizin» für zukünftige Allgemeinpraktiker (II)

Am Samstag, 8. November 2008, findet von 9 bis 16 Uhr im Ärztehaus FMT an der Freiestrasse 138 in Zürich der 20. Zürcher Workshop «Allgemeinmedizin» statt. Die Veranstaltung, die dieses Jahr in erster Auflage bereits im Juni durchgeführt wurde, richtet sich an zukünftige Allgemeinmediziner/innen mit Praxistätigkeit.

Programm

$9.00 \quad$ Kaffee und Gipfeli

9.15 Begrüssung, Organisation

Einführung: Dr. P. Landolt

Workshops (Rotation nach jeweils 75 Minuten)

9.45-11.00 Block I Qualitätssicherung, Diagnostik: Dr. P. Landolt

11.00-11.20 Pause

11.20-12.40 Block II Zusammenarbeit mit Spezialisten, Hausbesuche, der Notfallbesuch, Spitex: Dr. E. Bandi

\subsection{0-13.30 Mittagspause}

13.30-14.45 Block III Suchtkrankheiten, chronischer Schmerz: Dr. D. Ackermann

15.00-16.00 Fragen über wichtige Gesetze: Dr. H. U. Späth Schlussstunde: alle Referenten

$16.00 \quad$ Ende des Workshops

Die Kosten inklusive Mittagessen und Pausenverpflegung belaufen sich auf 250 Franken. Anmeldung an simone.ilic@yahoo.de oder Tel. 0435363751.

\section{Chargenrückruf}

Ephedrin HCl Amino, Injektionslösung (angemeldet unter Art. 95 HMG), Charge 4021/9

In einer Packung zu 10 Ampullen à $1 \mathrm{ml}$ der Charge 4021/9 des Präparates Ephedrin $\mathrm{HCl}$ Amino, Injektionslösung, wurde eine Ampulle à $1 \mathrm{ml}$ des Präparates Papaverin $\mathrm{HCl}$ Amino, Injektionslösung, gefunden. Letztere ist richtig als Papaverin $\mathrm{HCl}$ Amino, Injektionslösung, angeschrieben.

Die Firma Amino AG hat deshalb die erwähnte Charge des Präparates vom Markt zurückgerufen.

Der Rückruf erfolgte mittels Firmenschreiben an die mit dem Präparat belieferten Kunden.

\section{Rappel de lots}

Ephedrin HCl Amino, solution injectable (préparation annoncée selon l'article 95 LPTh), lot 4021/9

Une boîte de 10 ampoules à $1 \mathrm{ml}$ du lot 4021/9 de la préparation Ephedrin $\mathrm{HCl}$ Amino, solution injectable, contient par erreur une ampoule à $1 \mathrm{ml}$ de la préparation Papaverin $\mathrm{HCl}$ Amino, solution injectable, sur laquelle apparaît cependant bien la désignation Papaverin $\mathrm{HCl}$ Amino, solution injectable.

Pour cette raison, la société Amino AG a retiré du marché le lot susmentionné de la préparation.

Ce retrait a été communiqué par circulaire aux clients ayant reçu livraison du produit.

Amino $A G$

\section{Swiss MD PhD Association (SMPA)}

\section{Einladung an alle Interessierten und SMPA-Mitglieder}

Die Schweizerische MD-PhD-Gesellschaft lädt alle Mitglieder zu ihrer ordentlichen Generalversammlung vom 17. Oktober 2008 in Bern ein. Zudem möchten wir auch dieses Jahr - unabhängig von einer Mitgliedschaft - alle Interessierten zum Rahmenprogramm der Generalversammlung einladen.

Dieses Jahr nehmen wir das Thema «Forschung im Spital» auf und haben dazu eine Auswahl von Forschern, «Finanzierern» und Forschungsinteressierten eingeladen, um den aktuellen Stand sowie die Zukunft der Forschung im Spital zu diskutieren: Ist die Spitzenforschung im Spital noch finanzierbar? Braucht es wirklich Mediziner, die sich der Grundlagenforschung widmen? Wie sieht die Zukunft des «translational research» aus? Ist es möglich, Grundlagenforschung und klinische Tätigkeit zu kombinieren? Haben wir die Infrastruktur? Brauchen wir mehr Start-up-Stipendien? Was ist das Ziel des MD-PhD-Programms? Die Vorträge werden in Deutsch gehalten werden, Diskussion in Deutsch, Französisch und Englisch. Die Tagung findet im Polikliniktrakt 2 (PKT2), D517, Inselspital, Bern, statt. Wir freuen uns auf eine rege Teilnahme aller interessierten Kreise.

Detaillierte Programminformationen und Anmeldung unter www.smpa.org.

Für den Vorstand:

Dr. Yara Banz und Dr. Laurence Feldmeyer 